\section{Submitted from BMJ Quality}

\title{
Acute kidney injury; improving the communication from secondary to primary care
}

\author{
Clemency Nye, Suzanna Lake
}

To cite: Nye C, Lake S. Acute kidney injury; improving the communication from secondary to primary care. BMJ Quality Improvement Reports 2017;6:u211147. w6661. doi:10.1136/ bmjquality.u211147.w6661

CrossMark

Gloucestershire NHS Foundation Trust

\section{ABSTRACT}

Acute kidney injury (AKI) is a common but preventable event in secondary care. It is known to be associated with poorer outcomes for the patient's future health. Patients therefore require specific after-care in the community following an AKI, both in the short and long term. However, information about an inpatient AKI is often not communicated to primary care at discharge. Only $11.0 \%$ of discharge summaries contained full information about an AKI (including stage of $\mathrm{AKI}$, changes to medications and follow-up required) in August 2015.

We aimed to improve communication about AKI on discharge summaries via implementation of a series of interventions between June 2015 and March 2016. A specific section was added to the discharge summary software to prompt inclusion of information regarding AKI. An automatic warning message was added later as an additional prompt. A programme of education was provided for the junior doctors. A ward-based campaign was rolled out using the animated character 'Ned the Nephron,' using posters, emails and screen savers. We also introduced an AKI warning sticker for drug charts, which reminds the discharging doctor that the patient has had an AKI during the admission.

Our primary outcome was the percentage of discharge summaries that had the AKI section completed, as this contained all the desired information, including stage of AKI and frequency of follow up blood tests in primary care. Monthly data collections showed that this gradually increased from $4.7 \%$ in September 2015 to $35.0 \%$ in January 2016. We expect further increases with the recent introduction of the drug chart sticker.

\section{PROBLEM}

This project was conducted across Gloucester Royal Hospital and Cheltenham General Hospital, two district general hospitals with a combined total of over 1000 inpatient beds. The project was undertaken by a core team of foundation 2 doctors, with support from the renal team and the Gloucester Safety and Quality Improvement Academy.

The problem was that information about an inpatient AKI was inconsistently and infrequently being communicated to primary care at discharge. Only $11.0 \%$ of discharge summaries audited in the trust in August 2015 contained full information about an AKI (including stage, changes to medications and follow-up required). Discharge summaries are completed by junior doctors - work with this target group suggested that this low completion rate was due to a combination of lack of awareness of the long-term significance of AKI and role of primary care in AKI prevention, and time pressures when completing discharge paperwork.

The aim of the project was to increase the proportion of discharge summaries across both hospital sites which contained complete information about an inpatient AKI to 30\% by April 2016.

\section{BACKGROUND}

Acute kidney injury (AKI) is a common but preventable event in secondary care. It is known to be associated with both short-term and long-term adverse outcomes for the patient. ${ }^{1}$ Long term outcomes can include increased risk of repeat AKI, higher risk of development of chronic kidney disease and greater chance of progression to end-stage renal failure, ${ }^{2}$ higher risk of future cardiovascular and cerebrovascular events, ${ }^{3}$ and increased all-cause mortality at 10 years. ${ }^{4}$

The crucial role of the primary care physician is increasingly being recognised in AKI, particularly in the prevention of future AKI events. ${ }^{5}$ Up to $30 \%$ of AKI events are preventable,${ }^{6}$ although the contribution made by optimal management in primary care has yet to be quantified. In order for primary care to play an effective role in AKI prevention, the general practitioner must be provided with the relevant information from secondary care following a patient's AKI. Patients also require specific after-care in the community following an AKI, both in the short and long term. This usually comprises follow up blood tests to ensure stable renal 
function in the short term, and longer term ongoing assessment of prescribed medications. In addition, the primary care physician must take into account the patient's increased risk in all the above areas in their future management.

\section{BASELINE MEASUREMENT}

Our primary outcome was the percentage of discharge summaries that had the specific AKI section completed, as this contained all the desired information, including stage of AKI, changes to medications and frequency of follow up blood tests in primary care. The AKI section was introduced in May 2015, and therefore the baseline use of the AKI section prior to this was $0 \%$.

The first data collection occurred in August 2015. A list of patients who had an AKI during August and were discharged during the same month was compiled by cross-referencing between a list of patients flagged for AKI by the pathology IT system and a hospital discharge database. 149 patient discharges in total were audited. $10.7 \%$ contained complete information about the AKI (all three points as above.) $31.6 \%$ contained incomplete information ( 1 or 2 points of information.) $57.7 \%$ of discharge summaries had no mention at all about the AKI. We repeated data collection each month for a total of six months.

\section{DESIGN}

We questioned a number of junior doctors at different stages of training about the reasons why they did not always complete the AKI section of the discharge summary and their ideas for improvement. We then analysed the problem using a driver diagram. Our primary drivers were: junior doctor education and engagement, the design of the discharge summary software itself and remembering that the patient has previously had an AKI during the admission when completing the summary. We therefore designed our interventions to target these three areas.

In order to improve junior doctor engagement, we held two teaching sessions for the F1 and F2 doctors, who were completing the majority of discharge summaries. We aimed to emphasise the significance of AKI and the importance of follow-up following an AKI in these teaching sessions. We also targeted the wider hospital community via a trust-wide email, ward posters and article in the trust newsletter Outline. We created an animated character, 'Ned the Nephron,' which was used to tie the campaign together and create a talking point.

We worked closely with the IT department to improve the design of the discharge summary software. A specific box for information relating to AKI, with drop-down fields for easy completion, was added to the software. This box was then gradually amended over the course of the project following feedback from junior doctors about its' usability. Finally, an automated pop-up reminder message was added, to prompt doctors to complete the AKI section before completing the summary.

Remembering that the patient has had an AKI was a problem because patients were often being discharged some time after the AKI event or after transfer between wards, and it was difficult for doctors to recall the AKI, particularly if the AKI had been present on admission. We therefore created an AKI drug chart sticker which was added to the front of the drug chart when an AKI was recognised, and served as an ongoing reminder of the AKI.

\section{STRATEGY}

PDSA 1: introduction of AKI section on discharge summary

The dedicated AKI section on the discharge summary software was introduced in May 2015. The first data collection cycle was in August 2015. Each cycle of data collection analysed between 130 and 220 patient discharges. Results from August showed a 10.7\% compliance with use of the AKI section, in patients known to have had an AKI during their admission. This revealed the need for further promotion and explanation of the AKI section to its users.

\section{PDSA 2: junior doctor education}

Two teaching sessions were run for junior doctors in October 2015. We focused on the importance of good communication to primary care about inpatient AKIs, and the need for long-term follow up care after an AKI. Data was collected at the end of November. The proportion of completed AKI sections was $11.1 \%$, which was no significant improvement compared to the $10.7 \%$ seen in August. We therefore considered a more direct approach to reminding doctors about the AKI section.

PDSA 3: changes to discharge summary software

In December 2015, an automated reminder message was added to the discharge summary software, to serve as an additional prompt to junior doctors. Data collection at the end of December showed an increase in proportion of AKI sections completed to $29.5 \%$. This suggested that the reminder was an effective method of increasing compliance.

PDSA 4: introduction of drug chart stickers

We began to introduce the AKI drug chart stickers to wards in January 2015. The compliance increased to $35 \%$ at the end of January 2015.

\section{RESULTS}

Monthly data collections showed that the use of the AKI section of the discharge summary (in patients who had had an AKI) gradually increased from 10.7\% in August 2015 to $35.0 \%$ in January 2016. This was a marked improvement. On an informal basis, the degree of awareness of AKI and the need for effective communication to primary care improved among the junior doctor 


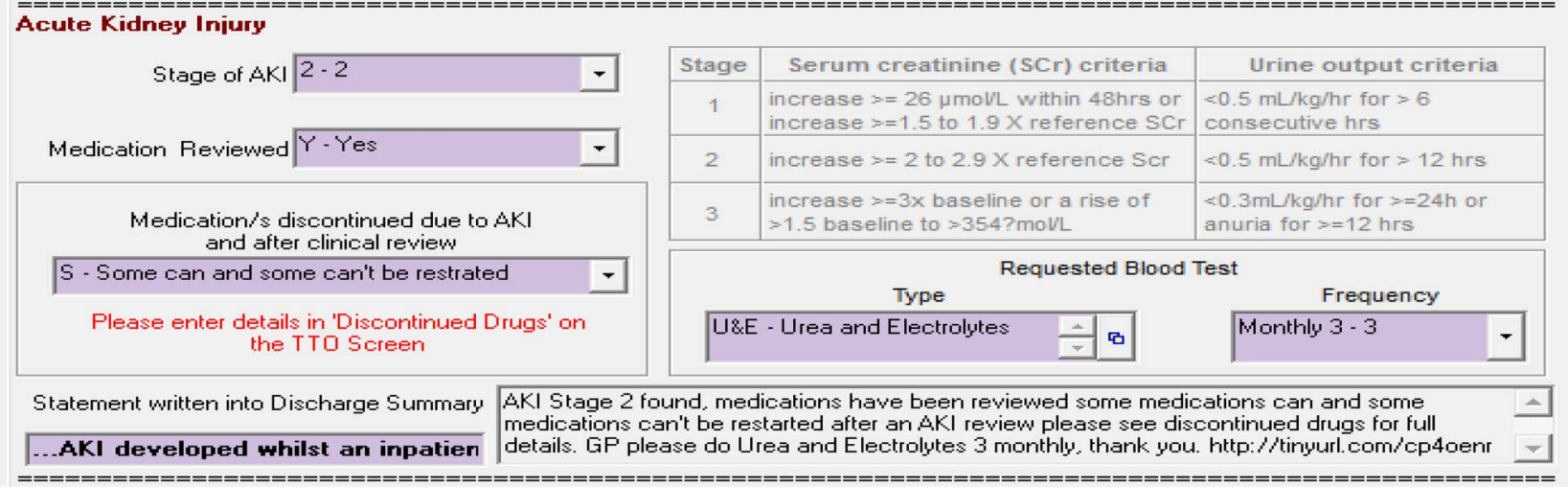

Figure 1

population. There is, however, the potential for further improvement, and for additional work on this subject, in particular examining how the information communicated from secondary care about an AKI is received and acted upon in primary care, and whether optimal follow up improves patient outcomes.

\section{LESSONS AND LIMITATIONS Lessons learned}

We have learnt that changing the behaviour of a population is a difficult process, and requires multiple approaches, and the involvement of a number of different key teams within the hospital. One of the main challenges we faced was being limited by the information systems software available within the trust, as the discharge summary software was unwieldy and difficult to use, and could not communicate with the pathology software.

It was interesting to note that a simple strategy of education for junior doctors was unsuccessful. We spoke to a number of junior doctors from different specialties and at different stages of training to try to understand the reasons behind this. We found that, although they had learnt some new information from the teaching, particularly regarding the effect of an AKI episode on a patient's long-term outcomes, they felt that they were already under pressure and could not easily take on a new requirement. It was also hard for them to associate filling in the AKI section with better patient outcomes, because the intended benefits to patients were long-term and happened outside of secondary care, and thus there was no visible benefit for them to appreciate.

\section{Limitations}

It was not possible to examine every discharge summary generated in the hospitals, so we randomly selected a sample to study from databases with which we were provided. We would like to have access to a wider sample in order to be sure that our random sample was representative of the whole population. In order to be sure that our findings are not the result of chance, we would also need to analyse more data and employ statistical tests.

\section{Balancing measures}

We did not test any balancing measures, but it would have been useful to examine measures such as length of time to complete discharge summary and junior doctor levels of satisfaction with the process. We could also work with general practitioners to assess whether they have found the information useful in their practice, and to what extent the additional recommendations e.g. follow up blood tests, have added to their workloads.

\section{Sustainability}

We have tried to make our changes sustainable in a number of ways. Firstly, we have ongoing support from the renal team and patient safety team, who remain invested in the project. The new cohort of foundation 1

Figure 2

\begin{tabular}{|c|c|c|c|}
\hline Aim & Primary Drivers & Secondary Drivers & Change Ideas \\
\hline \multirow{3}{*}{$\begin{array}{l}30 \% \text { of } \\
\text { patients to } \\
\text { have AKI box } \\
\text { completed by } \\
\text { April } 2016\end{array}$} & $\begin{array}{l}\text { Recognition that } \\
\text { patient has had AKI }\end{array}$ & Documentation & $\begin{array}{l}\longrightarrow \text { Drug chart stickers } \\
\longrightarrow \text { Date on sticker }\end{array}$ \\
\hline & $\begin{array}{l}\text { Junior doctor } \\
\text { engagement }\end{array}$ & $\begin{array}{l}\text { Education } \\
\text { Reminders }\end{array}$ & $\begin{array}{l}\longrightarrow \text { Teaching } \\
\longrightarrow \text { Emails, posters }\end{array}$ \\
\hline & $\begin{array}{l}\text { Discharge } \\
\text { summary } \\
\text { software }\end{array}$ & AKI box & $\longrightarrow$ Reminder alert box \\
\hline
\end{tabular}


Figure 3

Run chart of change in percentage of AKI sections completed over time

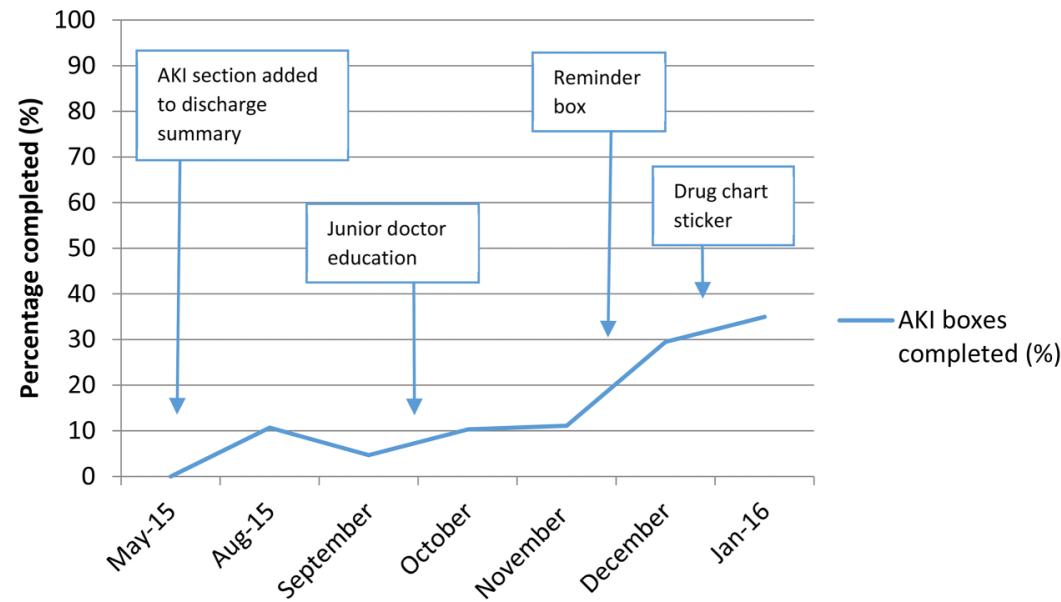

doctors in August 2016 have been thoroughly educated in the use of the AKI section. We have also given advice on the design of an AKI section in a new discharge summary software, which is due to be launched in the trust within the next year.

\section{Generalisability}

We feel that our project could be extended to other NHS trusts, as all hospitals have to generate discharge summaries to communicate from secondary to primary care, and communication about inpatient AKI events is

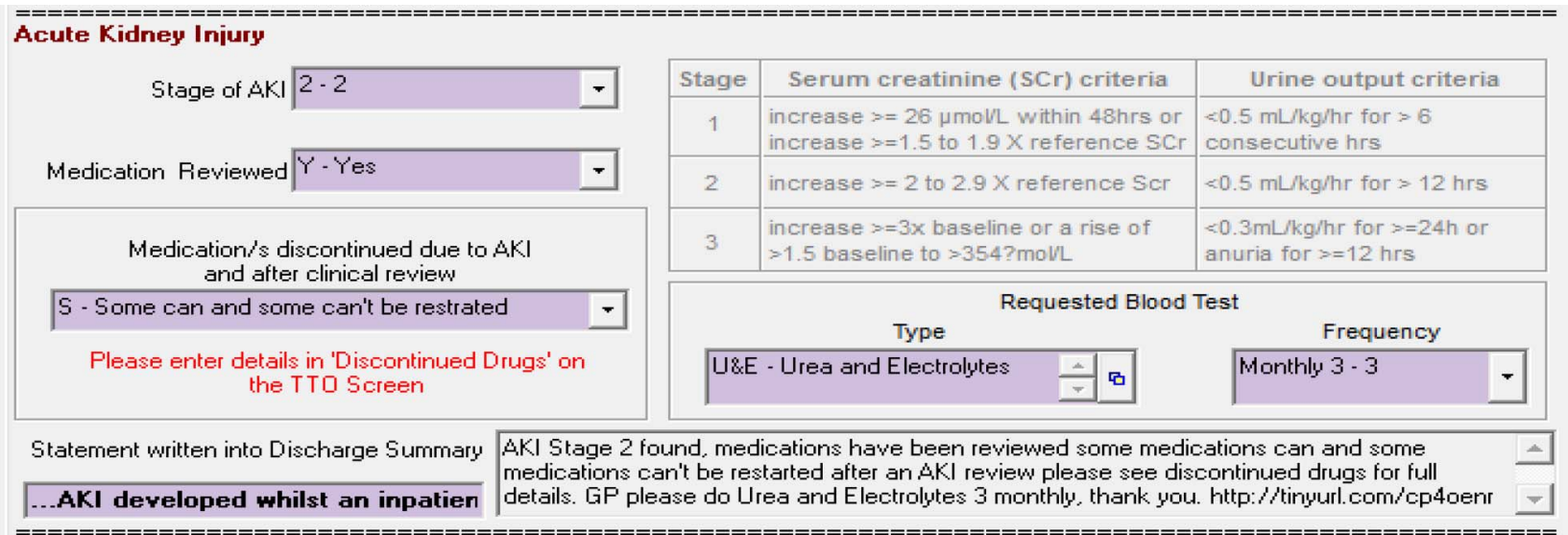

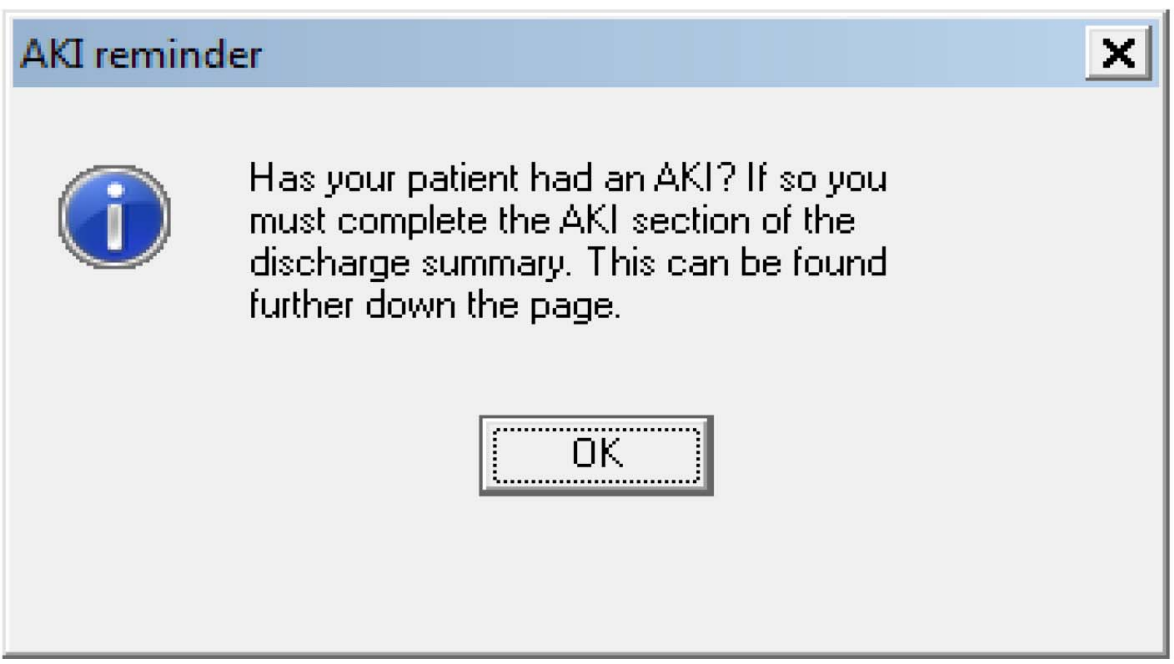

Figure 4 
being increasingly recognised as important. The project was run with similar results in two hospitals with different ranges of specialties and different catchment populations. The main difference between our trust and other trusts will be the computer software available, which may be more or less advanced in other trusts, or integrated with other software, such as pathology reporting software, to varying degrees.

\section{Cost effectiveness}

The burden of AKI is estimated to cost the NHS over $£ 1$ billion per year, or around $1 \%$ of the total NHS budget ${ }^{7}$. Good follow up care in the community after an AKI is hoped to reduce future AKIs and reduce re-admission rates. This should lead to cost savings for the trust. The project was also part of a national CQUIN, and therefore improving the information on discharge summaries was associated with a direct financial reward to the trust.

\section{CONCLUSION}

The problem of effective communication from secondary to primary care is common throughout hospital trusts. This is particularly the case for the subject of AKI, especially with recent focus on the importance of the role of primary care in AKI. ${ }^{5}$ We believe that our interventions could be easily implemented in other hospitals.

The series of interventions introduced led to a significant improvement in practice, and further improvement is expected with ongoing changes. Further data collection will be undertaken in late 2016, to assess the sustainability of the change.
Acknowledgements Additional core team member - Sophie Boyd Dr Preetham Bodanna - renal consultant and project mentor Gloucester Safety and Quality Improvement Academy - particularly Andrew Seaton (Director of Patient Safety), Jan Joseph and Alexandra Purcell Israr Baig - renal pharmacist

Declaration of interests Nothing to declare.

Ethical approval Ethics review was not sought because the study met criteria for exemption from such review, according to an institutional policy, as the work was deemed an improvement activity.

Open Access This is an open-access article distributed under the terms of the Creative Commons Attribution Non-commercial License, which permits use, distribution, and reproduction in any medium, provided the original work is properly cited, the use is non commercial and is otherwise in compliance with the license. See:

- $\mathrm{http}: / /$ creativecommons.org/licenses/by-nc/2.0/

- http://creativecommons.org/licenses/by-nc/2.0/legalcode

\section{REFERENCES}

1. Doyle J, Forni L. Acute kidney injury: short-term and long-term effects. Critical Care 2016;20:188.

2. Coca SG, Yusuf B, Shlipak MG, Garg AX, Parikh CR. Long-term risk of mortality and other adverse outcomes after acute kidney injury: a systematic review and meta-analysis. Am J Kidney Dis 2009;53:961-73.

3. Wu VC, Wu CH, Huang TM, Wang CY, Lai CF, Shiao CC, et al. Long-term risk of coronary events after AKI. J Am Soc Nephrol 2014;25:595-605.

4. Linder A, Fjell C, Levin A, Walley KR, Russell JA, Boyd JH. Small acute increases in serum creatinine are associated with decreased long-term survival in the critically ill. Am J Respir Crit Care Med 2014;189:1075-81.

5. Blakeman T, Harding S, O'Donoghue D. Acute kidney injury in the community: why primary care has an important role. Br J Gen Pract 2013;63:173-174.

6. NCEPOD. National Confidential Enquiry into Patient Outcome and Death. Acute kidney injury: adding insult to injury. 2009.

7. Kerr M, Bedford M, Matthews B, O'Donoghue D. The economic impact of acute kidney injury in England. Nephrology Dialysis Transplantation 2014;29:1362-8. 